T. O. Banakh, Department of Mathematics, Lviv National University, Lviv, Ukraine, and Instytut Matematyki, Akademia Świętokrzyska, Kielce, Poland. email: tbanakh@yahoo.com

O. V. Maslyuchenko, Department of Mathematical Analysis, Chernivtsi State University, vul. Kotsyubyńskoho 2, Chernivtsi, 58000, Ukraine. email: mathan@chnu.cv.ua

V. V. Mykhaylyuk, Department of Mathematical Analysis, Chernivtsi State University, vul. Kotsyubyńskoho 2, Chernivtsi, 58000, Ukraine.

email: vmasl@chnu.cv.ua

\title{
DISCONTINUOUS SEPARATELY CONTINUOUS FUNCTIONS AND NEAR COHERENCE OF P-FILTERS
}

\begin{abstract}
We prove that the problem of the existence of a discontinuous separately continuous function $f: X \times Y \rightarrow \mathbb{R}$ for any non-discrete Tychonov spaces $X, Y$ of countable pseudocharacter is equivalent to NCPF (Near Coherence of $P$-filters) which is independent of ZFC. Also for every nondiscrete Tychonov space $X$ we find an abelian topological group $G$ of countable cellularity and a discontinuous separately continuous function $f: X \times G \rightarrow \mathbb{R}$.
\end{abstract}

\section{Introduction.}

All calculus students learn that a function of two real variables $(x, y)$ can be continuous for each fixed $x$ and for each fixed $y$ without being continuous as a function of two real variables. The standard example illustrating this

Key Words: Separately continuous function, discontinuity point, near coherent filters, P-filter

Mathematical Reviews subject classification: 54C05, 54C08, 54A35, 03E35,03E50, 03E65

Received by the editors April 8, 2006

Communicated by: Krzysztof Chris Ciesielski 
phenomenon is the function $s p$ given by

$$
s p(x, y)= \begin{cases}\frac{2 x y}{x^{2}+y^{2}} & \text { if }(x, y) \neq(0,0) \\ 0 & \text { if }(x, y)=(0,0)\end{cases}
$$

It is clear that $s p$ is continuous as a function from the plane $\mathbb{R}^{2}$ to the real line $\mathbb{R}$ everywhere except for the origin.

Looking at this standard example, one could suggest that any non-discrete Tychonov spaces $X, Y$ admit a discontinuous separately continuous function $f: X \times Y \rightarrow \mathbb{R}$. However, that is not true. The following theorem proven in $[H W, 6.14]$ supplies us with many counterexamples. We recall that a topological space $X$ is called a $P$-space if each $G_{\delta}$-subset of $X$ is open.

Theorem 1. [HW] Every separately continuous function $f: X \times Y \rightarrow \mathbb{R}$ defined on the product of a $P$-space $X$ and a locally separable space $Y$ is continuous.

On the other hand, we also have a positive result proved in [MMMS, 2.6].

Theorem 2. [MMMS] Suppose $X, Y$ are Tychonov spaces with non-isolated $G_{\delta}$-points $a \in X, b \in Y$. If $Y$ is first countable or locally connected at the point $b$, then there is a separately continuous function $f: X \times Y \rightarrow \mathbb{R}$ continuous everywhere except for the point $(a, b)$. Moreover, this function $f$ can be chosen of the form $f=s p \circ(g \times h)$, where $g: X \rightarrow \mathbb{R}, h: Y \rightarrow \mathbb{R}$ are suitable continuous functions.

In the compact case we have the following characterization from $\left[\mathrm{My}_{2}\right]$.

Theorem 3. $[\mathbf{M}]$ Let $X, Y$ be compact Hausdorff spaces with non-isolated points $a \in X, b \in Y$. There exists a separately continuous function $f: X \times$ $Y \rightarrow \mathbb{R}$ with a unique discontinuity point $(a, b)$ if and only if there are sequences $\left(U_{n}\right)_{n \in \omega}$ and $\left(V_{n}\right)_{n \in \omega}$ of open non-empty subsets in $X, Y$ that converge to the points $a, b$, respectively.

The preceding theorems show that one should impose some restrictions on the nature of non-isolated points $a \in X, b \in Y$ to guarantee the existence of a separately continuous function $f: X \times Y \rightarrow \mathbb{R}$ discontinuous at $(a, b)$. In light of this, we can ask

Question 1. Suppose $X, Y$ are Tychonov spaces with non-isolated $G_{\delta}$-points $a \in X, b \in Y$. Is there a separately continuous function $f: X \times Y \rightarrow \mathbb{R}$ discontinuous at $(a, b)$ ? Can such a function $f$ be chosen of the form $f=$ $s p \circ(g \times h)$ for suitable continuous functions $g: X \rightarrow \mathbb{R}, h: Y \rightarrow \mathbb{R}$ ? 
This question is closely connected with the problem of relationship between various topologies on products of topological spaces, which appear naturally in the theory of separately continuous functions, see $[\mathrm{HW}],\left[\mathrm{My}_{1}\right]$, [HK]. One such natural topology on the product $X \times Y$ of two topological spaces is the cross topology $\gamma$, i.e., the maximal topology coinciding with the product topology on each "line" $\{x\} \times Y, X \times\{y\}$ where $x \in X$ and $y \in Y$. Another one is the topology $\sigma$ of separate continuity, i.e., the minimal topology on $X \times Y$ for which all separately continuous functions $X \times Y \rightarrow \mathbb{R}$ are continuous. It is known that $\tau \subset \sigma \subset \gamma$ for every Tychonov spaces $X, Y$, where $\tau$ stands for the usual product topology on $X \times Y$. Now the question appears: for which Tychonov spaces are these inclusions strict? According to Corollary 6.15 of [HW] the equality $(X \times Y, \sigma)=(X \times Y, \tau)$ holds for any $P$-space $X$ and any locally separable space $Y$. Thus the following question appears naturally.

Question 2. Is $(X \times Y, \sigma) \neq(X \times Y, \tau)$ for any Tychonov spaces $X, Y$ which are not $P$-spaces?

Let us remark that a topological space $X$ is a $P$-space if each point $x \in X$ is a $P$-point in $X$ (the latter means that each $G_{\delta}$-set $G \subset X$ containing the point $x$ is a neighborhood of $x$ in $X$ ). If $x \in X$ is a non-isolated $P$-point, then the pseudocharacter, tightness and $\pi$-character at $x$ are all uncountable.

Trying to answer Questions 1 and 2, we discovered (to our big surprise) that this can not be done in ZFC. Under Martin's Axiom these questions have negative answers. On the other hand, there are models of ZFC in which answers to these questions are affirmative. To describe these models we need to recall the notion of near coherence of filters introduced and studied in [Bl], [BS].

Two filters $\mathcal{F}_{1}, \mathcal{F}_{2}$ on a set $S$ are said to be near coherent if there is a finite-to-one function $h: S \rightarrow S$ such that $h\left(F_{1}\right) \cap h\left(F_{2}\right) \neq \emptyset$ for any $F_{1} \in \mathcal{F}_{1}$, $F_{2} \in \mathcal{F}_{2}$. A function $h: S \rightarrow S$ is said to be finite-to-one if $h^{-1}(s)$ is finite for every $s \in S$. We recall that a filter on a set $S$ is a collection $\mathcal{F} \not \supset \emptyset$ of subsets of $S$, closed with respect to supersets and intersections. If $\bigcap_{F \in \mathcal{F}} F=\emptyset$, then the filter $\mathcal{F}$ is called free. Next, $\mathcal{F}$ is called a $P$-filter if every countable collection $\mathcal{C} \subset \mathcal{F}$ has a pseudointersection in $\mathcal{F}$, i.e., a set $A \in \mathcal{F}$ such that $A \backslash C$ is finite for every $C \in \mathcal{C}$.

The following theorem reduces the problem of the existence of discontinuous separately continuous functions to the near coherence of $P$-filters.

Theorem 4. The following statements are equivalent.

1. For any Tychonov spaces $X, Y$ with non-isolated $G_{\delta}$-points $a \in X, b \in Y$ there are continuous maps $g: X \rightarrow \mathbb{R}, h: Y \rightarrow \mathbb{R}$ and a function $\psi: \mathbb{R} \times \mathbb{R} \rightarrow \mathbb{R}$ such that the function $f=\psi \circ(g \times h): X \times Y \rightarrow \mathbb{R}$ 
is separately continuous, discontinuous at $(a, b)$ and continuous at other points of $X \times Y$.

2. For any countable spaces $X, Y$ with a unique non-isolated point there is a discontinuous separately continuous function $f: X \times Y \rightarrow \mathbb{R}$.

3. $(X \times Y, \gamma) \neq(X \times Y, \tau)$ for any countable spaces $X, Y$ with a unique non-isolated point.

4. Any two free P-filters on a countable set are near coherent.

The last statement of Theorem 4 will be abbreviated as NCPF (Near Coherence of $P$-Filters) by analogy with NCF (Near Coherence of Filters) introduced and studied in [Bl]. We recall that NCF means that any two free filters on a countable set are near coherent. NCF contradicts Martin's Axiom but holds in some models of ZFC, see [BS] or [HM, p.100]. The following theorem describes the relationship between NCPF, NCF, Martin's Axiom, the Continuum Hypothesis and the small cardinals $\mathfrak{d}$ (the dominating number) and $\mathfrak{t}$ (the tower number), see [vD] or [Va].

Theorem 5. $(\mathrm{NCF}) \Rightarrow(\mathrm{NCPF}) \Rightarrow(\mathfrak{t} \neq \mathfrak{d}) \Rightarrow(\neg \mathrm{MA}) \Rightarrow(\neg \mathrm{CH})$.

Since both $\mathrm{NCF}$ and $\mathrm{CH}$ are independent of ZFC, we get that $\mathrm{NCPF}$ as well as all the equivalent statements of Theorem 4 are independent of ZFC.

We do not know if NCPF is strictly stronger than NCF.

Problem 6. Is there a model of ZFC in which NCPF holds but NCF fails? In particular, does NCPF hold in the Shelah's model [Wi] containing no P-point?

In this respect it is interesting to notice that assuming $\mathrm{NCF}$ in place of NCPF allows us to construct discontinuous separately continuous functions of some special type and take the function $\psi$ from Theorem 4(1) equal to the standard separately continuous function $s p$, defined at the beginning of the paper.

Theorem 7. Under NCF, for any Tychonov spaces $X, Y$ with non-P-points $a \in X, b \in Y$, there exist continuous functions $g: X \rightarrow \mathbb{R}$ and $h: Y \rightarrow \mathbb{R}$ such that the separately continuous function $f=s p \circ(g \times h): X \times Y \rightarrow \mathbb{R}$ is discontinuous at $(a, b)$. If in addition $\{a\}$ and $\{b\}$ are $G_{\delta}$-sets, then one can make $f$ continuous at all points other than $(a, b)$.

This theorem implies

Corollary 8. Assume NCF. If each separately continuous function $f: X \times$ $Y \rightarrow \mathbb{R}$ defined on the product of two Tychonov spaces $X, Y$ is continuous, then one of these spaces is a P-space. 
Problem 9. Are Theorem 7 and Corollary 8 true under NCPF?

According to Corollary 8 , it is consistent to assume that any Tychonov non- $P$-spaces $X, Y$ admit a discontinuous separately continuous function $f$ : $X \times Y \rightarrow \mathbb{R}$. As Theorem HW shows, the situation changes if one of the spaces $X$ or $Y$ is a $P$-space and the other is locally separable. It is natural to ask if the local separability in this theorem can be replaced by the countable cellularity. We shall show that this cannot be done.

We recall that a topological space $X$ has countable cellularity if any collection of pairwise disjoint non-empty open subsets of $X$ is at most countable. Among the most important examples of topological spaces with countable cellularity are the $\sigma$-compact topological groups and their subgroups (which admit an inner characterization as $\sigma$-bounded groups); see [ $\left.\mathrm{Tk}_{2}\right]$. We recall that a topological group $G$ is called $\sigma$-bounded if it is a countable union of totally bounded subsets (a subset $A \subset G$ is totally bounded if for every nonempty open set $U \subset G$ there is a finite subset $F \subset G$ with $A \subset(F \cdot U) \cap(U \cdot F))$. According to Tkachenko's Theorem [ $\left.\mathrm{Tk}_{1}\right]$, each $\sigma$-bounded topological group (being a dense subgroup of a $\sigma$-compact group) has countable cellularity.

Our last theorem shows that the local separability of the space $Y$ in Theorem HW cannot be replaced by the countable cellularity of $Y$.

Theorem 10. For any non-discrete Tychonov space $X$ there is a $\sigma$-bounded abelian topological group $G$ and a discontinuous separately continuous function $h: X \times G \rightarrow \mathbb{R}$.

\section{Proof of Theorem 10.}

The proof of Theorem 10 relies on the following

Lemma 2.1. For every non-discrete Tychonov (P-)space $X$ there exists a Tychonov (P-)space $Y$ with a unique non-isolated point and a bounded discontinuous separately continuous function $f: X \times Y \rightarrow \mathbb{R}$.

Proof. The space $X$, being non-discrete, contains a non-isolated point $a \in X$. Let $\tau(a)$ be the set of all neighborhoods of the point $a$ in $X$. For any $U \in \tau(a)$ let $\downarrow U=\{V \in \tau(a): V \subset U\}$. Let $b \notin \tau(a)$ be any point. On the union $Y=\{b\} \cup \tau(a)$ consider the topology in which all points $y \in \tau(a)$ are isolated while the sets $\{b\} \cup(\downarrow U), U \in \tau(a)$, are neighborhoods of the point $b$. Clearly, if $X$ is a $P$-space, then so is the space $Y$.

Next, we define a discontinuous separately continuous function $f: X \times$ $Y \rightarrow[0,1]$. For every neighborhood $U \in \tau(a)$ of $a$, fix a continuous function 
$f_{U}: X \rightarrow[0,1]$ such that $\max _{x \in X} f_{U}(x)=1$ and $f_{U}(\{a\} \cup(X \backslash U)) \subset\{0\}$. Define a function $f: X \times Y \rightarrow[0,1]$ by the formula

$$
f(x, y)= \begin{cases}0 & \text { if } y=b \\ f_{U}(x) & \text { if } y=U \in \tau(a)\end{cases}
$$

It is easy to see that the function $f$ is separately continuous but discontinuous at the point $(a, b)$.

Proof of Theorem 10. Given a non-discrete Tychonov space $X$ let $f: X \times$ $Y \rightarrow \mathbb{R}$ be a bounded discontinuous separately continuous function provided by Lemma 2.1. For every $x \in X$ let $\beta f_{x}: \beta Y \rightarrow \mathbb{R}$ be the continuous extension of the bounded continuous function $f_{x}: Y \rightarrow \mathbb{R}, f_{x}: y \mapsto f(x, y)$, onto the Stone-Čech compactification $\beta Y$ of $Y$. Next, let $A(\beta Y) \supset \beta Y$ be a free abelian topological group of $\beta Y$ (see [Ma]) and $h_{x}: A(\beta Y) \rightarrow \mathbb{R}$ be a unique continuous group homomorphism extending the function $\beta f_{x}$. Let $G$ be the group hull of the set $Y$ in $A(\beta Y)$. It is clear that $G$, being a subgroup of the $\sigma$-compact group $A(\beta Y)$, is a $\sigma$-bounded group and each element $g \in G$ can be written as $g=k_{1} y_{1}+\cdots+k_{n} y_{n}$ for some $n \in \mathbb{N}, k_{1}, \ldots, k_{n} \in \mathbb{Z}$, and $y_{1}, \ldots, y_{n} \in Y$. Finally, consider the function $h: X \times G \rightarrow \mathbb{R}$ defined by $h(x, g)=h_{x}(g)$.

It is clear that for every fixed $x \in X$ the function $h(x, \cdot): G \rightarrow \mathbb{R}$ is a continuous group homomorphism. To see that $h(\cdot, g): X \rightarrow \mathbb{R}$ is continuous for every fixed $g \in G$, observe that

$$
h(x, g)=h_{x}(g)=k_{1} f_{x}\left(y_{1}\right)+\cdots+k_{n} f_{x}\left(y_{n}\right)=k_{1} f\left(x, y_{1}\right)+\cdots+k_{n} f\left(x, y_{n}\right),
$$

where $g=k_{1} y_{1}+\cdots+k_{n} y_{n}$.

Therefore, $h: X \times G \rightarrow \mathbb{R}$ is a separately continuous function. Since $h \mid X \times Y=f$, the function $h$ is discontinuous.

\section{Proof of Theorem 7.}

Let $X_{1}, X_{2}$ be Tychonov spaces with non-P- $\left(G_{\delta^{-}}\right)$points $a_{i} \in X_{i}$ for $i=1,2$. Assuming NCF we have to find continuous maps $f_{i}: X_{i} \rightarrow \mathbb{R}$ for $i=1,2$ such that the separately continuous function $f=s p \circ\left(f_{1} \times f_{2}\right): X_{1} \times X_{2} \rightarrow \mathbb{R}$ is discontinuous at $\left(a_{1}, a_{2}\right)$ (and continuous at other points of $\left.X_{1} \times X_{2}\right)$.

For every $i=1,2$ fix an $F_{\sigma}$-subset $F_{i} \subset X_{i}$ with $a_{i} \in \bar{F}_{i} \backslash F_{i}$ and a continuous function $\alpha_{i}: X_{i} \rightarrow[0,1]$ such that $\alpha_{i}\left(a_{i}\right)=0$ and $\alpha_{i}\left(F_{i}\right) \subset(0,1]$. Let $B_{i}=\alpha_{i}^{-1}(0)$. Since $a \in \bar{F}_{i}$ and $F_{i} \cap B_{i}=\emptyset$, we conclude that $B_{i}$ is not a neighborhood of $a_{i}$ in $X_{i}$. If $a_{i}$ is a $G_{\delta}$-point of $X_{i}$, then we may take $F_{i}=X_{i} \backslash\left\{a_{i}\right\}$ and get that $B_{i}=\left\{a_{i}\right\}$. 
Now consider the separately continuous function $\operatorname{sp} \circ\left(\alpha_{1} \times \alpha_{2}\right): X_{1} \times X_{2} \rightarrow$ $\mathbb{R}$. It is easy to see that it is continuous on the set $X_{1} \times X_{2} \backslash B_{1} \times B_{2}$.

If this function is discontinuous at the point $\left(a_{1}, a_{2}\right)$, then we finish the proof. This is so if for some $i=1,2$ the image $\alpha_{i}(W)$ of any neighborhood $W \subset X_{i}$ of $a_{i}$ is a neighborhood of zero in $[0,1]$.

Next, consider the (non-trivial) case when $s p \circ\left(\alpha_{1} \times \alpha_{2}\right)$ is continuous at $\left(a_{1}, a_{2}\right)$ and for every $i \in\{1,2\}$ there is a neighborhood $W_{i} \subset X_{i}$ of the point $a_{i}$ whose image $\alpha_{i}\left(W_{i}\right)$ is not a neighborhood of zero in $[0,1]$. This means that $\alpha_{i}\left(W_{i}\right)$ contains many "holes" tending to zero. Using this fact, construct a continuous map $r_{i}: \alpha_{i}\left(W_{i}\right) \rightarrow S_{0}$ of $\alpha_{i}\left(W_{i}\right)$ onto the convergent sequence $S_{0}=\{0\} \cup S$ where $S=\left\{\frac{1}{n}: n \in \mathbb{N}\right\}$. This map may be chosen so that $r_{i}^{-1}(0)=\{0\}$. Observe that the family

$$
\mathcal{F}_{i}=\left\{S \cap r_{i} \circ \alpha_{i}(U): U \subset W_{i} \text { is a neighborhood of } a_{i}\right\}
$$

is a free filter on the countable set $S$.

NCF implies that the filters $\mathcal{F}_{1}$ and $\mathcal{F}_{2}$ are near coherent. Hence there is a finite-to-one function $\beta: S \rightarrow S$ such that $\beta\left(A_{1}\right) \cap \beta\left(A_{2}\right) \neq \emptyset$ for any $A_{i} \in \mathcal{F}_{i}$, $i=1,2$. The function $\beta$ admits a unique continuous extension $\bar{\beta}: S_{0} \rightarrow S_{0}$ with $\bar{\beta}(0)=0$. For every $i=1,2$, pick a continuous function $\lambda_{i}: X_{i} \rightarrow[0,1]$ such that $\lambda_{i}\left(X_{i} \backslash W_{i}\right)=\{0\}$ while $\lambda_{i}^{-1}(1)$ is a neighborhood of $a_{i}$ in $X_{i}$. Define a continuous function $f_{i}: X_{i} \rightarrow[0,1]$ letting

$$
f_{i}(x)= \begin{cases}\lambda_{i}(x) \cdot \bar{\beta} \circ r_{i} \circ \alpha_{i}(x)+\left(1-\lambda_{i}(x)\right) \alpha_{i}(x) & \text { if } x \in W_{i}, \\ \alpha_{i}(x) & \text { if } x \notin W_{i} .\end{cases}
$$

We claim that the separately continuous function $f=\operatorname{sp} \circ\left(f_{1} \times f_{2}\right): X_{1} \times X_{2} \rightarrow$ $\mathbb{R}$ is discontinuous at $\left(a_{1}, a_{2}\right)$ and continuous on the set $X_{1} \times X_{2} \backslash B_{1} \times B_{2}$.

The continuity of $f$ on the set $X_{1} \times X_{2} \backslash B_{1} \times B_{2}$ follows from the continuity of $s p$ on $\mathbb{R}^{2} \backslash\{(0,0)\}$ and the inclusions $f_{i}\left(X_{i} \backslash B_{i}\right) \subset(0,1]$ for $i=1,2$.

Assuming that $f$ is continuous at $\left(a_{1}, a_{2}\right)$, we may find neighborhoods $U_{i} \subset \lambda_{i}^{-1}(1) \subset W_{i}$ of the points $a_{i}, i=1,2$ such that $f\left(U_{1} \times U_{2}\right) \subset(-1,1)$. Let $A_{i}=S \cap r_{i} \circ \alpha_{i}\left(U_{i}\right) \in \mathcal{F}_{i}$ for $i=1,2$. Since $\beta\left(A_{1}\right) \cap \beta\left(A_{2}\right) \ni y$ for some $y \in S$, there exist points $x_{i} \in U_{i}, i=1,2$, such that $y=\beta \circ r \circ \alpha_{i}\left(x_{i}\right)$ for $i=1,2$ which yields that $f\left(x_{1}, x_{2}\right)=s p(y, y)=1$, a contradiction with $f\left(x_{1}, x_{2}\right) \in f\left(U_{1} \times U_{2}\right) \subset(-1,1)$.

\section{Proof of Theorem 4.}

To prove Theorem 4 we shall verify the implications $(1) \Rightarrow(2) \Rightarrow(3) \Rightarrow(4) \Rightarrow$ (1). In fact, the implications $(1) \Rightarrow(2) \Rightarrow(3)$ are trivial. 
To prove the implication $(4) \Rightarrow(1)$, repeat the proof of Theorem 7 ; to handle the case in which one of the filters $\mathcal{F}_{1}$ or $\mathcal{F}_{2}$ fails to be a $P$-filter, so that $\mathcal{F}_{1}$ and $\mathcal{F}_{2}$ fail to be nearly coherent, apply the following additional lemma (using the same denotation as in the proof of Theorem 7).

Lemma 4.1. If $B_{1}=\left\{a_{1}\right\}$ and $\mathcal{F}_{1}$ is not a $P$-filter, then there is a function $\psi: \mathbb{R} \times \mathbb{R} \rightarrow \mathbb{R}$ and continuous maps $f_{i}: X_{i} \rightarrow[0,1]$ for $i=1,2$ such that the function $f=\psi \circ\left(f_{1} \times f_{2}\right): X_{1} \times X_{2} \rightarrow \mathbb{R}$ is separately continuous, discontinuous at $\left(a_{1}, a_{2}\right)$ and continuous on the set $X_{1} \times X_{2} \backslash B_{1} \times B_{2}$.

Proof. Since $\mathcal{F}_{1}$ is not a $P$-filter, there is a sequence $\left(A_{n}\right)_{n=1}^{\infty} \subset \mathcal{F}_{1}$ having no pseudointersection in $\mathcal{F}_{1}$. Replacing each set $A_{n}$ by $A_{1} \cap \cdots \cap A_{n}$, we may assume that the sequence $\left(A_{n}\right)_{n=1}^{\infty}$ is decreasing. Let $\mathbb{R}_{0}$ stand for the real line endowed with the topology $\tau$ coinciding with the usual topology of the real line at all points except for 0 . A subset $U \subset \mathbb{R}$ is declared a neighborhood of 0 in the topology $\tau$ if $U \backslash\{0\}$ is open in $\mathbb{R}$ and $U \supset A_{n} \cup\{0\}$ for some $n \in \mathbb{N}$.

It is easy to construct a separately continuous function $\psi: \mathbb{R}_{0} \times \mathbb{R} \rightarrow \mathbb{R}$ which is continuous on the set $\mathbb{R}_{0} \times \mathbb{R} \backslash\{(0,0)\}$ and satisfies the condition

$$
\psi(x, y)= \begin{cases}1 & \text { if } 0<x \leq y=\frac{1}{n} \text { and } x \notin A_{n} \text { for some } n \in \mathbb{N} \\ 0 & \text { otherwise }\end{cases}
$$

for any $(x, y) \in S_{0} \times S_{0}$.

For every $i=1,2$, pick a continuous function $\lambda_{i}: X_{i} \rightarrow[0,1]$ such that $\lambda_{i}\left(X_{i} \backslash W_{i}\right)=\{0\}$ and $\lambda_{i}^{-1}(1)$ is a neighborhood of $a_{i}$ in $X_{i}$. Next, for every $i=1,2$ consider the continuous function $f_{i}: X_{i} \rightarrow[0,1]$ defined by

$$
f_{i}(x)= \begin{cases}\lambda_{i}(x) \cdot r_{i} \circ \alpha_{i}(x)+\left(1-\lambda_{i}(x)\right) \alpha_{i}(x) & \text { if } x \in W_{i}, \\ \alpha_{i}(x) & \text { if } x \notin W_{i} .\end{cases}
$$

Observe that $f_{2}^{-1}(0)=B_{2}, f_{1}^{-1}(0)=B_{1}=\left\{a_{1}\right\}$, and $f_{1}$ is continuous as a function from $X_{1}$ into $\mathbb{R}_{0}$. Then the function $f=\psi \circ\left(f_{1} \times f_{2}\right): X_{1} \times X_{2} \rightarrow \mathbb{R}$ is separately continuous and continuous outside the set $B_{1} \times B_{2}=\left(f_{1} \times\right.$ $\left.f_{2}\right)^{-1}(0,0)$.

It remains to verify that $f$ is discontinuous at the point $\left(a_{1}, a_{2}\right)$. Assuming the converse we would find a neighborhood $U_{1} \times U_{2} \subset \lambda_{1}^{-1}(1) \times \lambda_{2}^{-1}(1)$ of $\left(a_{1}, a_{2}\right)$ in $X_{1} \times X_{2}$ such that $f\left(U_{1} \times U_{2}\right) \subset(-1,1)$. Let $P=S \cap r_{1} \circ \alpha_{1}\left(U_{1}\right)$. Since $P \in \mathcal{F}_{1}, P$ is not a pseudointersection of the collection $\left\{A_{n}\right\}_{n=1}^{\infty}$, and thus $P \backslash A_{n}$ is infinite for some $n \in \mathbb{N}$. Since the sequence $\left(A_{n}\right)_{n=1}^{\infty}$ is decreasing, $P \backslash A_{m}$ is infinite for all $m \geq n$. Pick any point $\frac{1}{m} \in r_{2} \circ \alpha_{2}\left(U_{2}\right)$ with $m \geq n$. Since $P \backslash A_{m}$ is infinite, we can find a point $\frac{1}{k} \in P \backslash A_{m}$ with $k \geq m$. By 
the definition of $\psi, \psi\left(\frac{1}{k}, \frac{1}{m}\right)=1$. Pick any points $x_{1} \in U_{1}, x_{2} \in U_{2}$ with $r_{1} \circ \alpha_{1}\left(x_{1}\right)=\frac{1}{k}$ and $r_{2} \circ \alpha_{2}\left(x_{2}\right)=\frac{1}{m}$. Then $f\left(x_{1}, x_{2}\right)=\psi\left(\frac{1}{k}, \frac{1}{m}\right)=1$, a contradiction which proves the discontinuity of $f$ at $\left(a_{1}, a_{2}\right)$.

This proves the implication $(4) \Rightarrow(1)$ of Theorem 4 . To prove the implication $(3) \Rightarrow(4)$ we need several auxiliary results.

We define a subset $A \subset \mathbb{N} \times \mathbb{N}$ to be cross-finite if for every $n \in \mathbb{N}$ the intersection $A \cap(\mathbb{N} \times\{n\} \cup\{n\} \times \mathbb{N})$ is finite. Under a standard cross-finite subset, we understand a subset $A \subset \mathbb{N} \times \mathbb{N}$ of the form $A=\bigcup_{n \in \mathbb{N}} h^{-1}(n) \times$ $h^{-1}(n)$ for some finite-to-one function $h: \mathbb{N} \rightarrow \mathbb{N}$.

Lemma 4.2. Every cross-finite subset $A \subset \mathbb{N} \times \mathbb{N}$ lies in the union of two standard cross-finite sets.

ProOF. Inductively, we can construct a strictly increasing function $f: \mathbb{N} \rightarrow \mathbb{N}$ such that $f(n)>n$ and $f(n) \geq \max \{k \in \mathbb{N}: \exists i \leq n$ with $(k, i) \in A$ or $(i, k) \in A\}$. Next, define recursively the function $g: \mathbb{N} \rightarrow \mathbb{N}$ letting $g(1)=f(1)$ and $g(n)=f(g(n-1))$ for $n>1$. Let also $g(-1)=g(0)=1$. Finally, define two finite-to-one functions $h_{1}, h_{2}: \mathbb{N} \rightarrow \mathbb{N}$ letting $h_{1}^{-1}(k)=[g(2 k-3), g(2 k-1))$ and $h_{2}^{-1}(k)=[g(2 k-2), g(2 k))$ for $k \in \mathbb{N}$. We claim that $A \subset A_{1} \cup A_{2}$, where $A_{i}=\bigcup_{k \in \mathbb{N}} h_{i}^{-1}(k) \times h_{i}^{-1}(k)$ for $i=1,2$. Indeed, fix any $(i, j) \in A$. Without loss of generality, $i \leq j$. Find $n \in \mathbb{N}$ with $g(n-1) \leq i<g(n)$. Then $g(n-1) \leq i \leq j \leq f(i)<f(g(n))=g(n+1)$ and $(i, j) \in[g(n-1), g(n+1))^{2} \subset$ $A_{1} \cup A_{2}$.

Lemma 4.3. Two filters $\mathcal{F}_{1}$ and $\mathcal{F}_{2}$ on $\mathbb{N}$ are near coherent if and only if there is a cross-finite subset $A \subset \mathbb{N} \times \mathbb{N}$ such that $A \cap\left(F_{1} \times F_{2}\right) \neq \emptyset$ for every $F_{i} \in \mathcal{F}_{i}, i=1,2$.

Proof. To prove the "only if" part, assume that the filters $\mathcal{F}_{1}$ and $\mathcal{F}_{2}$ are near coherent. Then there is a finite-to-one function $h: \mathbb{N} \rightarrow \mathbb{N}$ such that $h\left(F_{1}\right) \cap h\left(F_{2}\right) \neq \emptyset$ for every sets $F_{i} \in \mathcal{F}_{i}, i=1,2$. Consider the standard crossfinite subset $A=\bigcup_{n \in \mathbb{N}} h^{-1}(n) \times h^{-1}(n)$ of $\mathbb{N} \times \mathbb{N}$. It follows that $\left(F_{1} \times F_{2}\right) \cap A \neq$ $\emptyset$ for every $F_{i} \in \mathcal{F}_{i}, i=1,2$.

To prove the "if" part, assume that $A$ is a cross-finite subset of $\mathbb{N} \times \mathbb{N}$ such that $\left(F_{1} \times F_{2}\right) \cap A \neq \emptyset$ for every $F_{1} \in \mathcal{F}_{1}$ and $F_{2} \in \mathcal{F}_{2}$. By Lemma 4.2 , there are two standard cross-finite subsets $A_{1}, A_{2}$ of $\mathbb{N}$ such that $A \subset A_{1} \cup A_{2}$. Find finite-to-one functions $h_{1}, h_{2}: \mathbb{N} \rightarrow \mathbb{N}$ with $A_{i}=\bigcup_{n \in \mathbb{N}} h_{i}^{-1}(n) \times h_{i}^{-1}(n)$ for $i=1,2$. Assuming that the filters $\mathcal{F}_{1}$ and $\mathcal{F}_{2}$ are not near coherent we would find sets $F_{1} \in \mathcal{F}_{1}$ and $F_{2} \in \mathcal{F}_{2}$ such that $h_{i}\left(F_{1}\right) \cap h_{i}\left(F_{2}\right)=\emptyset$ for every $i=1,2$. This yields $\left(F_{1} \times F_{2}\right) \times\left(A_{1} \cup A_{2}\right)=\emptyset$, a contradiction with $A \subset A_{1} \cup A_{2}$ and $\left(F_{1} \times F_{2}\right) \cap A \neq \emptyset$. 
Now we are able to prove the implication $(3) \Rightarrow(4)$. Given a free filter $\mathcal{F}$ on $\mathbb{N}$ let $\mathbb{N}_{\mathcal{F}}=\mathbb{N} \cup\{\infty\}$ be the one-point extension of the discrete space $\mathbb{N}$ with the sets $\{\infty\} \cup F, F \in \mathcal{F}$, being neighborhoods of the unique non-isolated point $\infty$ of $\mathbb{N}_{\mathcal{F}}$.

Lemma 4.4. Two free P-filters $\mathcal{F}_{1}$ and $\mathcal{F}_{2}$ on $\mathbb{N}$ are near coherent if and only if the cross topology $\gamma$ on $\mathbb{N}_{\mathcal{F}_{1}} \times \mathbb{N}_{\mathcal{F}_{2}}$ is strictly stronger than the product topology.

Proof. If free filters $\mathcal{F}_{1}$ and $\mathcal{F}_{2}$ on $\mathbb{N}$ are near coherent, then by Lemma 4.3, there is a cross-finite set $A \subset \mathbb{N} \times \mathbb{N}$ such that $\left(F_{1} \times F_{2}\right) \cap A \neq \emptyset$ for every $F_{i} \in \mathcal{F}_{i}, i=1,2$. It is clear that the set $U=\left(\mathbb{N}_{\mathcal{F}_{1}} \times \mathbb{N}_{\mathcal{F}_{2}}\right) \backslash A$, being a neighborhood of $(\infty, \infty)$ in the cross topology, is not a neighborhood of $(\infty, \infty)$ in the product topology. Thus the cross topology in strictly stronger than the product topology on $\mathbb{N}_{\mathcal{F}_{1}} \times \mathbb{N}_{\mathcal{F}_{2}}$.

Next, we prove that the cross topology on $\mathbb{N}_{\mathcal{F}_{1}} \times \mathbb{N}_{\mathcal{F}_{2}}$ coincides with the product topology if the free $P$-filters $\mathcal{F}_{1}$ and $\mathcal{F}_{2}$ are not near coherent. In fact, it suffices to verify that these topologies coincide at $(\infty, \infty)$. Let $U \subset$ $\mathbb{N}_{\mathcal{F}_{1}} \times \mathbb{N}_{\mathcal{F}_{2}}$ be a neighborhood of $(\infty, \infty)$ in the cross topology. Since $\mathcal{F}_{1}, \mathcal{F}_{2}$ are $P$-filters, we may find sets $P_{1} \in \mathcal{F}_{1}, P_{2} \in \mathcal{F}_{2}$ such that $\left(P_{1} \times\{j\}\right) \backslash U$ and $\left(\{i\} \times P_{2}\right) \backslash U$ are finite for every $i, j \in \mathbb{N}$ with $(\infty, j),(i, \infty) \in U$. Moreover, we can assume that $P_{1} \times\{\infty\} \cup\{\infty\} \times P_{2} \subset U$. Then the set $A=\left(P_{1} \times P_{2}\right) \backslash U$ is cross-finite. By Lemma 4.3, there are sets $F_{1} \in \mathcal{F}_{1}, F_{2} \in \mathcal{F}_{2}$ such that $\left(F_{1} \times F_{2}\right) \cap A=\emptyset$. Replacing $F_{i}$ by $F_{i} \cap P_{i}$, if necessary, we may assume that $F_{i} \subset P_{i}$ for $i=1,2$. Then $F_{1} \times F_{2} \subset U$ and $\left(\{\infty\} \cup F_{1}\right) \times\left(\{\infty\} \cup F_{2}\right) \subset U$ which shows that $U$ is a neighborhood of $(\infty, \infty)$ in the product topology of $\mathbb{N}_{\mathcal{F}_{1}} \times \mathbb{N}_{\mathcal{F}_{2}}$.

\section{Proof of Theorem 5.}

First we recall some standard notation, see [vD], [Va]. We identify cardinals with the smallest ordinals of the corresponding size. It is well known that each ordinal $\alpha$ can be uniquely written as $\alpha=\beta+n$, where $\beta$ is a limit ordinal and $n \in \omega$ (such $n$ will be called the integer part of $\alpha$ and will be denoted by $n(\alpha))$.

Let $\omega$ stand for the set of finite ordinals, $[\omega]^{\omega}$ denote the set of all infinite subsets of $\omega$ and ${ }^{\omega} \omega$ be the set of all functions from $\omega$ into $\omega$. On ${ }^{\omega} \omega$ we consider the usual partial order: $f \leq g$ iff $f(n) \leq g(n)$ for all $n \in \omega$. A set $D \subset{ }^{\omega} \omega$ is dominating if for every $f \in{ }^{\omega} \omega$ there is $g \in D$ with $f \leq g$.

For $A, B$ in $[\omega]^{\omega}$ we say that $A$ is almost included in $B$ (denoted $A \subset^{*} B$ ) if $A \backslash B$ is finite. A set $A \subset \omega$ is called a pseudo-intersection of a family $\mathcal{T} \subset[\omega]^{\omega}$ 
if $A \subset^{*} B$ for every $B \in \mathcal{T}$. A family $\mathcal{T} \subset[\omega]^{\omega}$ well-ordered by $\supset^{*}$ is called a (decreasing) scale. By a normal scale we shall mean a family $\mathcal{T} \subset[\omega]^{\omega}$ for which there are an ordinal $\alpha$ and a map $f: \alpha \rightarrow \mathcal{T}$ such that $f(\beta) \subset^{*} f(\gamma)$, $f(\gamma+1) \subset f(\gamma)$, and $f(\beta) \not \ngtr n(\beta)$ for any ordinals $\gamma<\beta<\alpha$. A family $\mathcal{T} \subset[\omega]^{\omega}$ is a tower if $\mathcal{T}$ is a scale having no infinite pseudo-intersection. Let

$$
\begin{aligned}
& \mathfrak{d}=\min \left\{|D|: D \text { is a dominating set in }{ }^{\omega} \omega\right\} \\
& \mathfrak{t}=\min \left\{|\mathcal{T}|: \mathcal{T} \subset[\omega]^{\omega} \text { is a tower }\right\}
\end{aligned}
$$

It is known that $\aleph_{1} \leq \mathfrak{t} \leq \mathfrak{d} \leq \mathfrak{c}$, where $\mathfrak{c}$ stands for the cardinality of continuum. It is consistent to assume that any of the above inequalities is strict, see [vD]. Martin's Axiom (MA) implies the equality $\mathfrak{t}=\mathfrak{d}=\mathfrak{c}$, see $[\mathrm{Ru}, 8]$ or [Va].

Among the implications $(\mathrm{NCF}) \Rightarrow(\mathrm{NCPF}) \Rightarrow(\mathfrak{d} \neq \mathfrak{t}) \Rightarrow(\neg \mathrm{MA}) \Rightarrow(\neg \mathrm{CH})$ of Theorem 5 , all except for the second one are trivial or well-known. The second implication will be proved next.

Lemma 5.1. If $\mathfrak{t}=\mathfrak{d}$, then there are two free $P$-filters $\mathcal{F}_{1}, \mathcal{F}_{2}$ on $\omega$ that are not near coherent.

Proof. Assume that $\mathfrak{t}=\mathfrak{d}$ and let $D=\left\{g_{\alpha}: \alpha<\mathfrak{d}\right\}$ be a dominating set in ${ }^{\omega} \omega$ with $g_{0} \equiv 0$. By transfinite induction we shall construct normal scales $\mathcal{T}_{1}=\left\{X_{\alpha}: \alpha<\mathfrak{d}\right\}$ and $\mathcal{T}_{2}=\left\{Y_{\alpha}: \alpha<\mathfrak{d}\right\}$ of subsets of $\omega$ such that for every ordinal $\alpha<\mathfrak{d}$ the following condition is satisfied:

$X_{\alpha} \cap Y_{\alpha}=\emptyset$ and $g_{\alpha}(x)<y$ for every $x, y \in X_{\alpha} \cup Y_{\alpha}$ with $x<y . \quad\left(*_{\alpha}\right)$

Let $X_{0}=\{2 n+1: n \in \omega\}$ and $Y_{0}=\{2 n+2: n \in \omega\}$. Assume that for some ordinal $\alpha<\mathfrak{d}$ we have constructed normal scales $\left\{X_{\beta}: \beta<\alpha\right\}$ and $\left\{Y_{\beta}: \beta<\alpha\right\}$ satisfying the conditions $\left(*_{\beta}\right)$ for all $\beta<\alpha$. Since $\alpha<\mathfrak{d}=\mathfrak{t}$, the scale $\left\{X_{\beta}: \beta<\alpha\right\}$ is not a tower and hence has an infinite pseudointersection $I_{1} \not \supset n(\alpha)$. In case of a non-limit ordinal $\alpha$, we may additionally assume that $I_{1} \subset X_{\alpha-1}$. The same argument allows us to find an infinite pseudo-intersection $I_{2}$ for the family $\left\{Y_{\beta}: \beta<\alpha\right\}$ such that $I_{2} \not \ngtr n(\alpha)$ and $I_{2} \subset Y_{\alpha-1}$ if $\alpha$ is not limit.

By induction, construct infinite disjoint subsets $X_{\alpha} \subset I_{1}$ and $Y_{\alpha} \subset I_{2}$ such that $g_{\alpha}(x)<y$ for any $x, y \in X_{\alpha} \cup Y_{\alpha}$ with $x<y$. This completes the inductive step.

For every $i=1,2$ consider the filter $\mathcal{F}_{i}=\left\{F \subset \omega: F \supset T\right.$ for some $\left.T \in \mathcal{T}_{i}\right\}$ whose base is the scale $\mathcal{T}_{i}$. Using the facts that the cofinality of the cardinal $\mathfrak{d}$ is uncountable $[\mathrm{vD}, 3.1]$ and $\mathcal{T}_{i}$ is a scale, we can easily show that $\mathcal{F}_{i}$ is a $P$-filter. Assuming that the $P$-filters $\mathcal{F}_{1}, \mathcal{F}_{2}$ are near coherent, we would find a finite-to-one function $h: \omega \rightarrow \omega$ such that $h\left(X_{\alpha}\right) \cap h\left(Y_{\alpha}\right) \neq \emptyset$ for every $\alpha<\mathfrak{d}$. Since the set $D$ is dominating, there is an ordinal $\alpha<\mathfrak{d}$ such that $g_{\alpha}(i) \geq \max \{k \in \omega: h(k)=h(i)\}$ for each $i \in \omega$. Since $h\left(X_{\alpha}\right) \cap h\left(Y_{\alpha}\right) \neq \emptyset$, 
there are $i \in X_{\alpha}$ and $j \in Y_{\alpha}$ with $h(i)=h(j)$. Since $X_{\alpha} \cap Y_{\alpha}=\emptyset$, we get $i \neq j$. If $i<j$, then by $\left(*_{\alpha}\right)$ we get $g_{\alpha}(i)<j$ and by the choice of $g_{\alpha}$, $j>g_{\alpha}(i) \geq \max \{k \in \omega: h(k)=h(i)\} \geq j$, a contradiction. So, $j<i$. In this case, we use $\left(*_{\alpha}\right)$ to get a contradiction: $i>g_{\alpha}(j) \geq i$.

\section{References}

[Bl] A. Blass, Near Coherence of Filters I: Cofinal Equivalence of Models of Arithmetic, Notre Dame J. Formal Logic, 27 (1986), 579-591.

[BS] A. Blass, S. Shelah, Near Coherence of Filters III: A Simplified Consistency Proof, Notre Dame J. Formal Logic, 30(4) (1989), 530-538.

[vD] E. K. van Douwen, The Integers and Topology, Handbook of SetTheoretic Topology (K. Kunen and J. E. Vaughan eds.), NorthHolland, Amsterdam, (1984), 111-167.

[HK] J. Hart, K. Kunen, On the Regularity of the Topology of Separate Continuity, Topology Appl., 123(1) (2002), 103-123.

[HM] K. P. Hart, J. van Mill, Open Problems on $\beta \omega$, Open Problems in Topology (J. van Mill and G. M. Reed eds.), North-Holland, Amsterdam, (1990), 97-125.

[HW] M. Henriksen, R. G. Woods, Separate Versus Joint Continuity: A Tale of Four Topologies, Topology Appl., 97 (1999), 175-205.

[Ma] A. A. Markov, On Free Topological Groups, Izv. AN SSSR. Ser. Mat., 9 (1945), 3-64.

[MMMS] V. K. Maslyuchenko, O. V. Maslyuchenko, V. V. Mykhaylyuk, O. V. Sobchuk, Paracompactness and Separately Continuous Mappings, General Topology in Banach Spaces (T. Banakh ed.), Nova Publ., NY, (2001), 147-169.

$\left[\mathrm{My}_{1}\right] \quad$ V. V. Mykhaylyuk, The Topology of Separate Continuity and a Generalization of Sierpinski Theorem, Matem. Studii, 14(2) (2000), 193-196.

$\left[\mathrm{My}_{2}\right]$ V. V. Mykhaylyuk, One-point Discontinuities of Separately Continuous Functions on the Product of Two Compact Spaces, Ukrain. Math. J., 57(1) (2005), 112-120; translated from Ukr. Mat. Zhurn., 57 (2005), 94-101. 
[Ru] M. E. Rudin, Martin's Axiom, Handbook of Mathematical Logic Vol. II (Set theory) (J. Barwise ed.), North-Holland, Amsterdam, (1982).

[Tk $\mathrm{Tk}_{1}$ M. Tkachenko, The Souslin Property in Free Topological Groups over Compact Spaces, Math. Notes, 34 (1983), 790-793.

$\left[\mathrm{Tk}_{2}\right] \quad$ M. Tkachenko, Introduction to Topological Groups, Topology Appl., 86 (1998), 179-231.

[Va] J. E. Vaughan, Small Uncountable Cardinals and Topology, Open Problems in Topology (J. van Mill and G. M. Reed eds), NorthHolland, Amsterdam, (1990), 195-216.

[Wi] E. Wimmers, The Shelah P-point Independence Theorem, Israel J. Math., 43 (1982), 28-48. 
348 T. O. Banakh, O. V. Maslyuchenko and V. V. Mykhaylyuk 